|| Print ISSN: 2589-7837 || Online ISSN: 2581-3935 ||

International Journal of Medical Science and Diagnosis Research (IJMSDR)

Available Online at www.ijmsdr.com

NLM (National Library of Medicine ID: 101738824)

\title{
A Case of Irritant Contact Dermatitis Mimicking as Localized Bullous Pemphigoid.
}

\author{
Dr A. Ramesh ${ }^{1}$, Dr Rajkumar Kannan ${ }^{2}$, Dr Samuel Jeyaraj Daniel ${ }^{3}$, \\ Dr M.Bhuvana ${ }^{4}$ \\ ${ }^{1}$ MD., DD., DNB (DVL), Professor, Department of DVL, Madras Medical College \\ ${ }^{2}$ MD DD, Associate Professor, Department of DVL, Madras Medical College \\ ${ }^{3} \mathrm{MD}$, Associate Professor, Department of DVL, Madras Medical College \\ ${ }^{4}$ MBBS, Postgraduate Student, Department of DVL, Madras Medical College
}

\section{Conflicts of Interest: Nil}

Corresponding author: Dr M.Bhuvana

DOI: https://doi.org/10.32553/ijmsdr.v5i8.843

\begin{abstract}
:
Introduction: 19 years old adult male a college going scholar presented to the Derm OPD, with complaints of multiple clear fluid filled lesions over left forearm around the hemodialysis fistula site. Patient gave history of applying thrombophob ointment over fistula site, which led to development of tense blisters filled with clear fluid that mimics localized bullous pemphigoid, where in appropriate and adequate investigations proved it to be a case of Irritant Contact Dermatitis. Thrombophob ointment which contains benzyl nicotinate and heparin applied over the hemodialysis fistula site leads to bullous lesions of Irritant Contact Dermatitis (ICD)which mimics as localized bullous pemphigoid over fistula site.
\end{abstract}

Keywords:_Hemodialysis fistula site,Irritant Contact Dermatitis, localized Bullous Pemphigoid.

\section{Case Report}

A 19 years old adult male a college going scholar presented to the Derm OPD, with complaints of multiple clear fluid filled lesions over left forearm around the hemodialysis fistula site .Patient is a known case of chronic kidney disease Grade -5 , for the past 4 years and on hemodialysis for the past 3 years. No history of preceding pruritic or urticarial lesions prior to the onset of fluid filled lesions. History of topical application of thrombophob ointment on and off for 3 months duration. History of frequent application of same ointment multiple times is present three days prior to the onset of bullous lesions..Patient gives history of clear fluid in the blister cavity which becomes hemorrhagic after each episode of hemodialysis (figure 3).No history of topical application of indigenous or native medications.

On Dermatological examination Multiple tense blisters of varying sizes ,the largest of which was measuring $5 \mathrm{~cm}$ in diameter filled with clear fluid were present over the extensor aspect of left forearm. Blisters were found over the normal looking skin around the hemodialysis fistula site (figure 1). Few vesicles with clear fluid were also seen over dorsum of left hand.( figure2)

Bulla spread sign (Lutz sign) shows rounded extension and Nikolskiy sign was negative .No other lesions elsewhere in the body. Scalp, hair, nails, mucosa, palms and soles were normal.

With this clinical picture a case of localized Bullous pemphigoid around the fistula site of 
hemodialysis was thought off and appropriate bed side and other relevant investigations like biopsy and Immunoflourescence were planned.

Tzanck smear showed, no acantholytic cells,rather tadpole cells( figure 4) which are in favour of Irritant Contact Dermatitis were present.Serum vitamin D levels were decreased(8.01 ng/ml) ( Table 1).Blister fluid D dimer and $\mathrm{P}$ - selectin levels were not done, because of the lockdown during covid-19 pandemic.Biopsy and DIF were done from vesicle and normal skin respectively after requesting nephrologist to do hemodialysis with out heparin to avoid bleeding during biopsy.

Interestingly biopsy shows massive spongiosis, multiple intraepidermal vesicles and single bulla cavity containing increased neutrophils, eosinophils and fibrin. Inflammatory infiltrates were seen in upper dermis.Exocytosis present (figure5) DIF did not show any Deposition of Immune Complexes in the Basement Membrane Zone (BMZ)( figure 6,7, 8).Patient was diagnosed as a case of irritant contact dermatitis to thrombophob ointment and treated with topical steroids and systemic antibiotics. Lesions healed completely with post inflammatory hypopigmentation. (figure 9)

\section{Discussion:}

Contact dermatitis is an inflammatory response of the skin to the various exogenous substances. It can be irritant contact dermatitis or allergic contact dermatitis. Irritant contact dermatitis is due to a nonspecific response of skin to release of various inflammatory cytokines. It depends on

1. Duration and concentration of substance used and frequency of exposure,

2. Type of skin i.e, preexisting atopic tendency/ atopic dermatitis or previously challenged skin,

3. Environmental factors including temperature and humidity.

Infants, females, and elderly individuals are at more risk to get irritant contact dermatitis.

Acute phase mainly presents with more burning sensation than itching and development of erythema, edema, oozing, vesicles and pustules with well demarcated borders around the area of contact and does not spread beyond that. Chronic phase shows lichenification.

Common allergens are colophony ,balsam of peru nickel, cobalt and fragrance mix, ${ }^{[1]},{ }^{[2]}$ Recently reported allergens are formaldehyde, ${ }^{[3]}$ and methyl dibromo glutaronitrile, ${ }^{[4]}$

Diagnosis is made by detailed clinical history, Dermatological examination, patch test and histopathology. Histopathology mainly shows spongiosis and inflammatory infiltrates. Drug induced bullous disorders are considered as common differential diagnosis. Irritant contact dermatitis is usually self limiting condition, can be treated with high potent topical corticosteroid and antihistamines.

Bullous Pemphigoid was considered as a idiopathic disorder, ${ }^{[5]}$ but recent data suggest various triggering factors, includes ultraviolet rays, medication, and skin injury. ${ }^{[6]}$ Localized BP has been reported in the clinical settings of radiotherapy, surgical wounds, trauma, or burns. ${ }^{[7]}$ And also in patients with end-stage renal disorder (ESRD) undergoing hemodialysis. ${ }^{[8]}$

Our patient is on hemodialysis for the past 3 years on and off and has every possibility to develop localized Bullous Pemphigoid around the hemodialysis fistula site. With this clinical picture the patient was provisionally diagnosed as a case of localized Bullous Pemphigoid around the fistula of hemodialysis,Hence we started working on the patient. To our great shock even though the clinical picture was compatible with localized Bullous Pemphigoid ,the biopsy findings showed massive spongiosis, multiple intraepidermal vesicles and single bulla cavity containing increased neutrophils, eosinophils and fibrin. Inflammatory infiltrates were seen in upper dermis. Exocytosis also seen. Direct immunoflourscence findings were not compatible with Bullous Pemphigoid. Also the lesions did not disseminate to the other areas of skin and was confined only to the extensor aspect of left forearm. Hence final diagnosis of irritant contact dermatitis was arrived. Patient was treated with 
saline soaks, topical corticosteroid and systemic antibiotics and advised strictly to stop the external application of thrombophob ointment. Lesions healed completely with post inflammatory hypo pigmentation. Also the patient has no recurrence of blisters following the initial episode and even after 4 months no lesions were noticed. This case is being reported for its interesting clinical presentation of Irritant Contact Dermatitis around hemodialysis fistula site that mimics as localized Bullous pemphigoid.

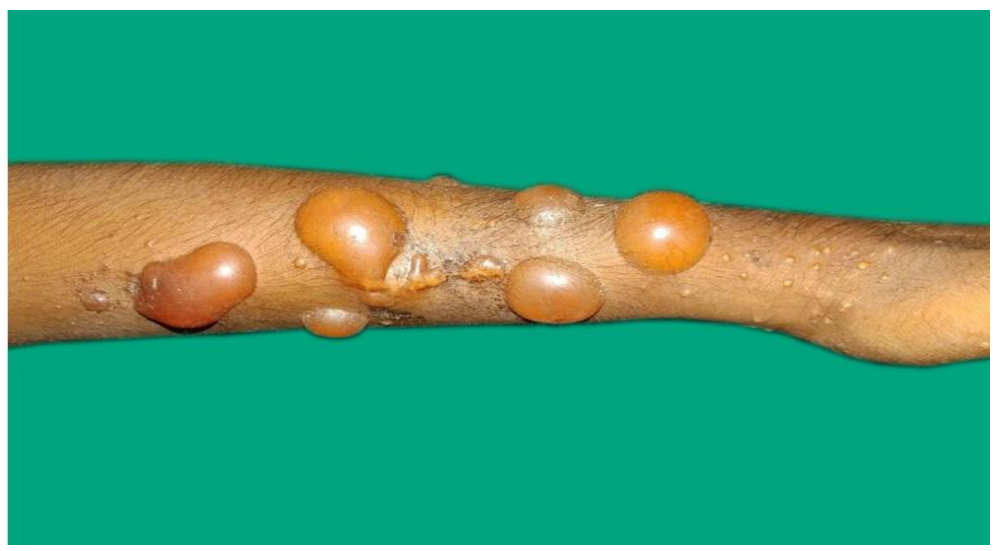

Figure1: Multiple clear fluid filled vesicles around hemodialysis fistula site of left forearm

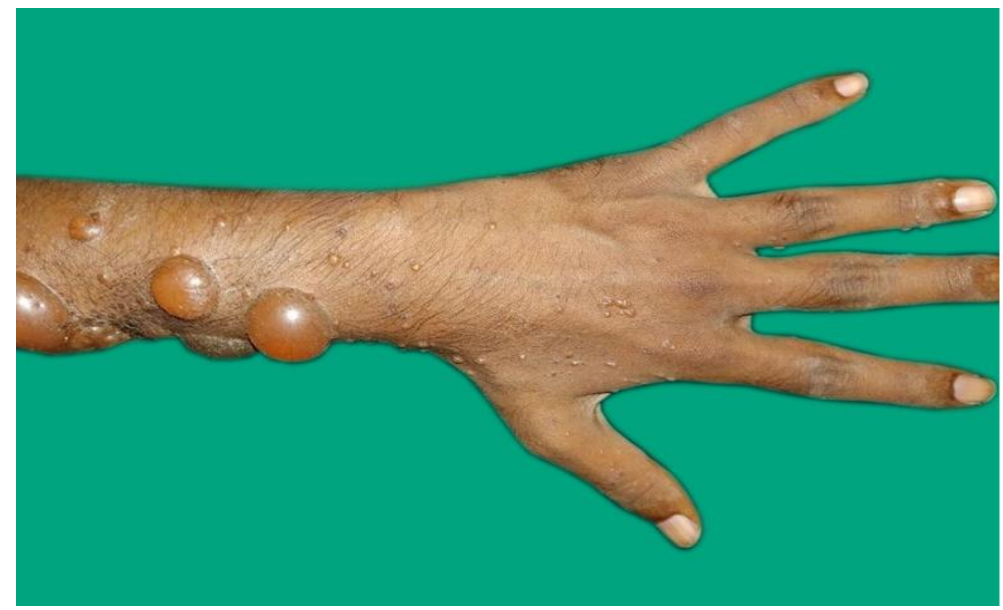

Figure 2: Few tiny vesicles present over dorsum of hand.

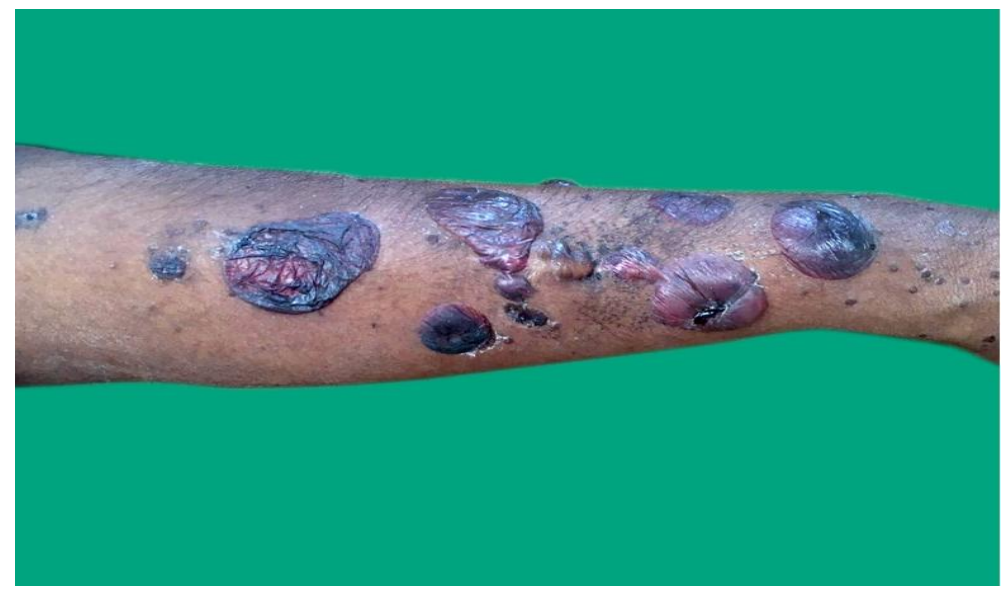

Figure 3: Fluid becomes hemorrhagic after hemodialysis episodes. 


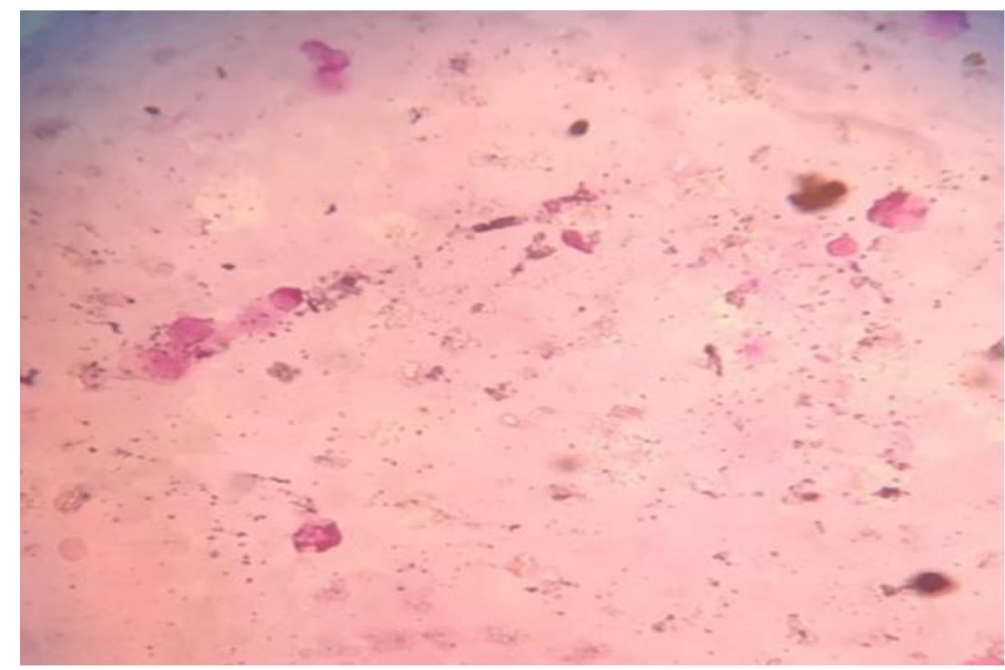

Figure 4: Tzanck smear shows no acntholytic cells and presence of tadpole cells.

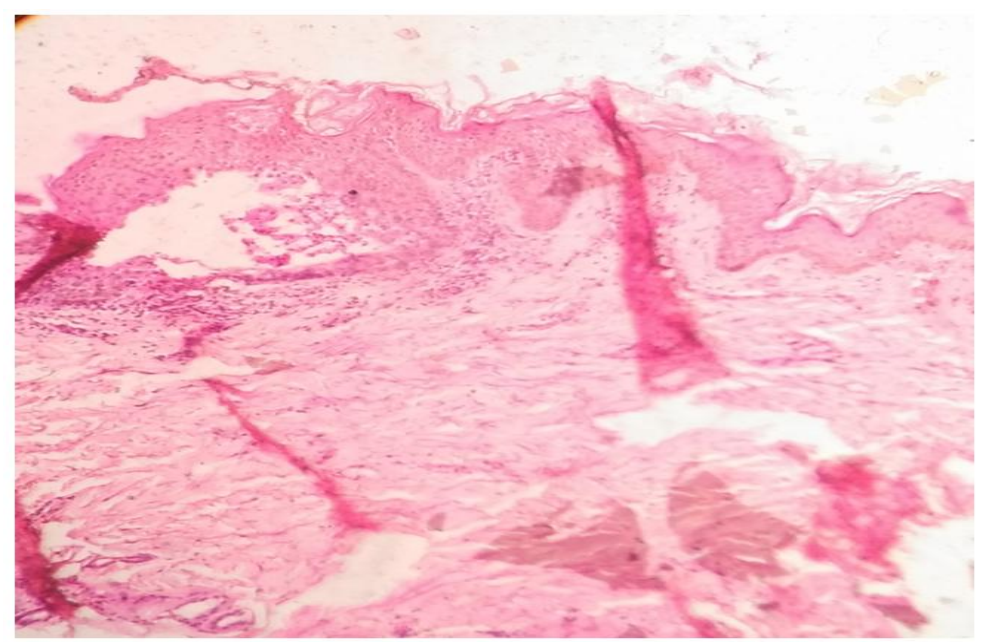

Figure 5: massive spongiosis, multiple intraepidermal vesicles and single bulla cavity containing increased neutrophils, eosinophils and fibrin. Inflammatory infiltrates were seen in upper dermis. Exocytosis also seen.

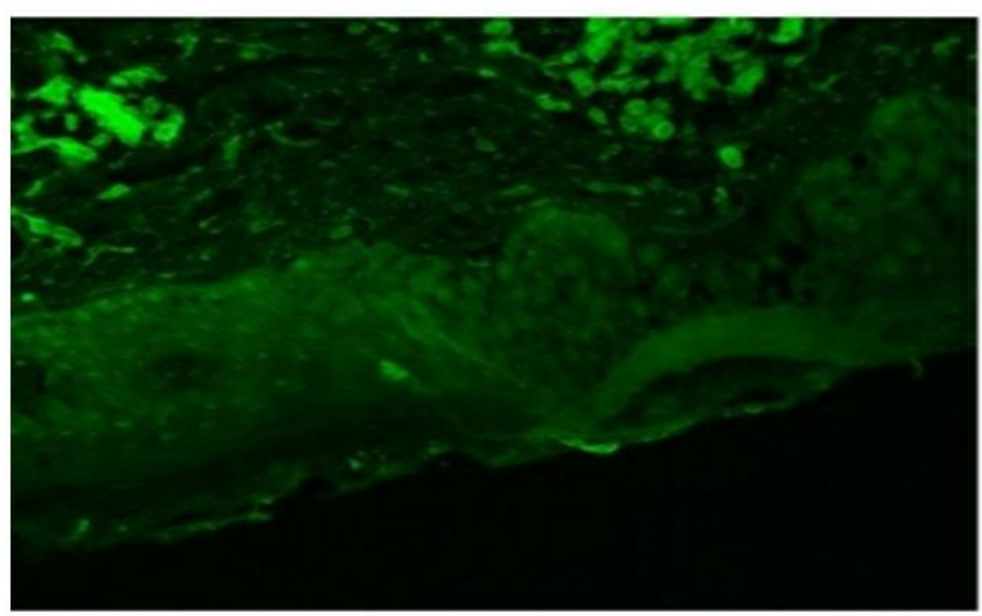

Figure 6: IgG negative 


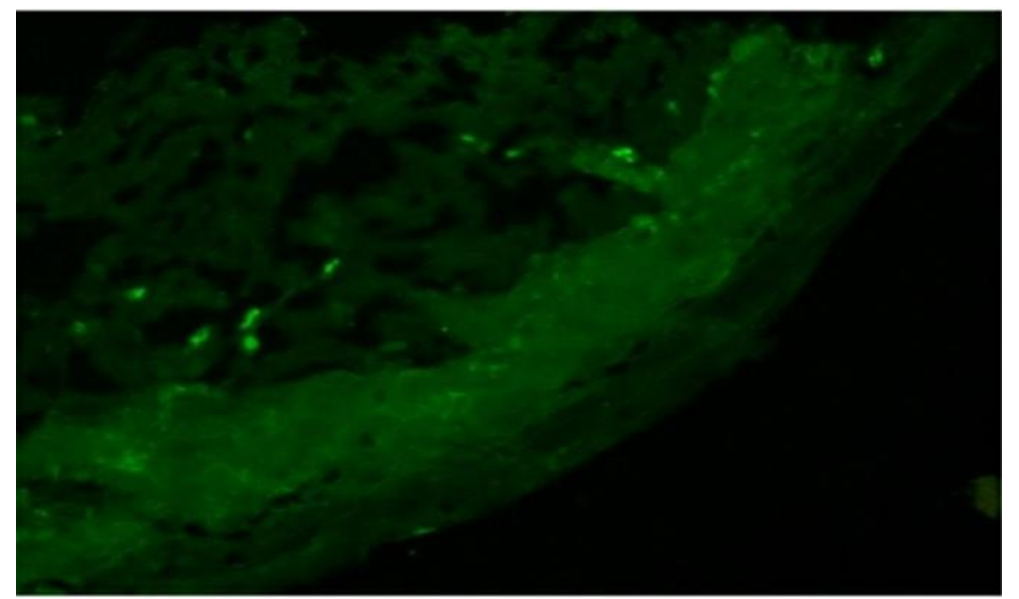

Figure 7: Complement 3 negative

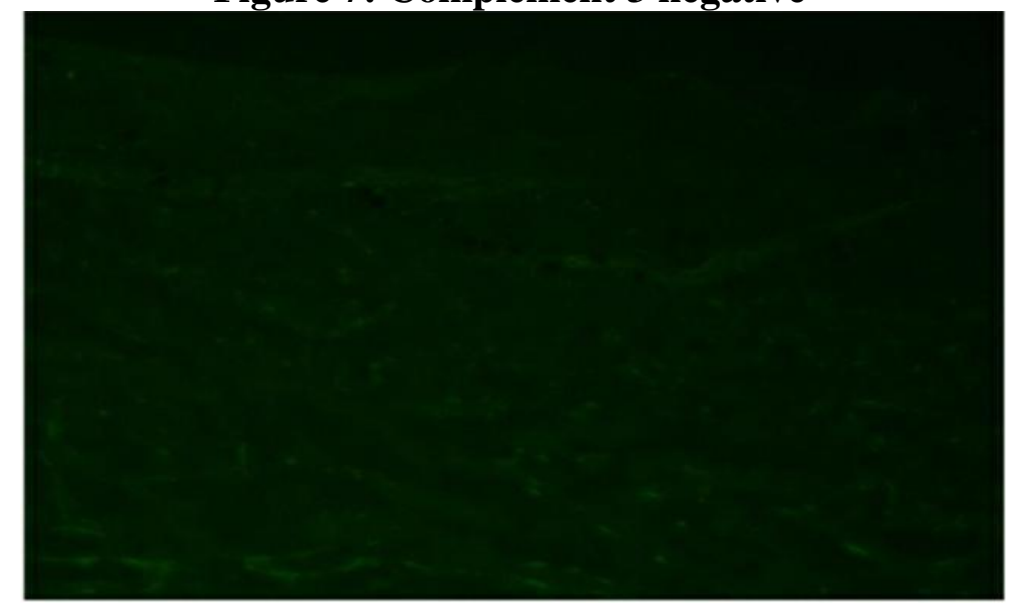

Figure 8: IgA negative

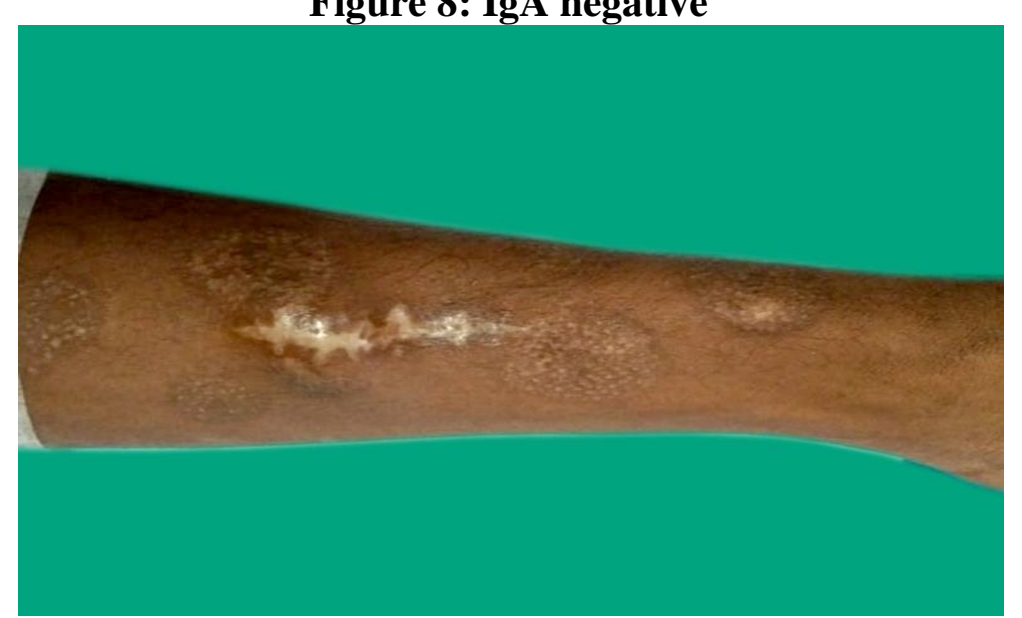

Figure 9: Lesions healed completely with post inflammatory hypo pigmentation

Table 1:

\begin{tabular}{|l|l|l|}
\hline Serial no & Substance & Normal value \\
\hline $\mathbf{1}$ & Serum vitamin D & $\mathbf{8 . 0 1} \mathbf{~ n g} / \mathbf{~ m l}$ \\
\hline
\end{tabular}




\section{REFERENCES:}

1. Meding B. Epidemiology of hand eczema in an industrial city. Acta Derm Venereol 1990;153(Supp 1):1-43.[Google Scholar]

2. Heydorn S, Johansen JD, Andersen KE, Bruze M, Svedman C, White I, et al. Fragrance allergy in patients with hand eczema. Contact Dermatitis 2003;48:31723.[Google Scholar]

3. Cronin E. Formaldehyde is a significant allergen in women with hand eczema. Contact Dermatitis 1991;25:276-82.[Google Scholar]

4. Zachariae C, Rastogi S, Devantier C, menne T, Johanses J. Methyl dibromo glutaronitrile: Clinical experience and exposure based risk assessment. Contact Dermatitis 2003;48:150-4. [Google Scholar]
5. Schmidt E, Zillikens D. Pemphigoid diseases. Lancet 2013;381:320-32

6. Taghipour K, Chi CC, Vincent A, Groves RW, Venning V, Wojnarowska $F$. The association of bullous pemphigoid with cerebrovascular disease and dementia: A case-control study. Arch Dermatol 2010;146:1251-4.

7. Pardo J, Rodrguez-Serna M, Mercader P, Fortea JM. Localized bullous pemphigoid overlying a fistula for hemodialysis. J Am Acad Dermatol 2004;51:S131-2.

8. Osipowicz K, Kalinska-Bienias A, Kowalewski C, Wozniak K. Development of bullous pemphigoid during the haemodialysis of a young man: Case report and literature survey. Int Wound $\mathbf{J}$ 2017; 14:288-92 